\title{
A genome-wide search for linkage to asthma phenotypes in the genetics of asthma international network families: evidence for a major susceptibility locus on chromosome $2 p$
}

Sreekumar G Pillai ${ }^{1}$, Mathias N Chiano ${ }^{1}$, Nicola J White ${ }^{1}$, Marcy Speer ${ }^{2}$, Kathleen C Barnes ${ }^{3,4}$, Karin Carlsen ${ }^{5}$, Jorrit Gerritsen ${ }^{6}$, Peter Helms ${ }^{7}$, Warren Lenney, Michael Silverman ${ }^{9}$, Peter Sly ${ }^{10}$, John Sundy ${ }^{11}$, John Tsanakas ${ }^{12}$, Andrea von Berg ${ }^{13}$, Moira Whyte ${ }^{14}$, Shela Varsani ${ }^{1}$, Paul Skelding ${ }^{1}$, Michael Hauser ${ }^{2}$, Jeffery Vance ${ }^{2}$, Margaret Pericak-Vance ${ }^{2}$, Daniel K Burns ${ }^{1}$, Lefkos T Middleton ${ }^{1}$, Shyama R Brewster ${ }^{1}$, Wayne $\mathrm{H}$ Anderson ${ }^{1}$ and John H Riley ${ }^{1}$

\footnotetext{
${ }^{1}$ Genetics Research Glaxo SmithKline, Research Triangle Park, NC, USA/Stevenage, UK; ${ }^{2}$ Center for Human Genetics, Duke University Medical Center, Durham, NC, USA; ${ }^{3}$ Department of Medicine, Johns Hopkins University, Baltimore, MD, USA; ${ }^{4}$ Department of Epidemiology, Johns Hopkins University, Baltimore, MD, USA; ${ }^{5}$ Ullevaal University Hospital, Oslo, Norway; ${ }^{6}$ Groningen University Hospital, Groningen, Netherlands; ${ }^{7}$ Department of Child Health, University of Aberdeen Royal Aberdeen Children's Hospital, Aberdeen, UK; ${ }^{8}$ Directorate of Child Health, Academic Department of Pediatrics, North Staffordshire Hospital, Stoke on Trent, UK; ${ }^{9}$ Division of Child Health, University of Leicester, Leicester, UK; ${ }^{10}$ Telethon Institute for Child Health Research, Center for Child Health Research, University of Western Australia, Perth, Australia; ${ }^{11}$ Duke University Medical Center, Durham, NC, USA; ${ }^{2}$ Pediatric Respiratory Unit, Pediatric Respiratory Medicine, Hippokration General Hospital, Thessaloniki, Greece; ${ }^{13}$ Abt. Fuer Kinderheilkunde Foschungsinstitut zur Praevention von Allergien und Atemwegserkrankungen im Kindesalter, Wesel, Germany; ${ }^{14}$ Academic Unit of Respiratory Medicine, University of Sheffield, Sheffield, UK
}

European Journal of Human Genetics (2007) 15, 714. doi:10.1038/sj.ejhg.5201840

Correction to: European Journal of Human Genetics (2006) 14, 307-316. doi:10.1038/sj.ejhg.5201532.
Owing to a typesetting error, the affiliations for the above paper were published incorrectly. The corrected list is now shown. 